\title{
AI for Advertising
}

\section{Maria Cristina ENACHE ${ }^{\star}$}

\begin{tabular}{l} 
A R T I C L E I N F O \\
\hline Article history: \\
Accepted April 2020 \\
Available online May 2020 \\
\hline JEL Classification \\
K22, M21 \\
Keywords: \\
IT, AI, Advertising \\
\hline
\end{tabular}

\begin{abstract}
A B S T R A C T
For marketing teams, finding and cultivating valuable customers is the key to building a profitable business. Leading companies know that personalized customer experiences and streamlined operations are essential to their success. Companies that extensively use AI ensure the benefits of increasing customer satisfaction and loyalty, while improving operations that add to their core level.
\end{abstract}

(C) 2020 EAI. All rights reserved.

\section{Introduction}

The advertising agencies are specialized in the realization of marketing services (creation of advertising spots, consulting in advertising problems, choice of media, etc.). At the end of the 19th century the advertising agencies worked for the owners of media companies. In the US the advertising agencies worked for the big publications selling the advertising space and receiving a commission instead. Later, besides the sellers of advertising space, the agencies also specialized in the actual writing of the advertising announcements that appeared in those spaces. Currently, their activity has diversified greatly, an advertising agency working simultaneously with a portfolio of companies, both in the image of promoting them, as well as for promoting the products, services offered by the company. Currently the owners of media channels offer the recognized advertising agencies a standard commission from the time / commercial space bought by them $(10-15 \%$ of the contract value).

The main advantages that come from using the services of an advertising agent are:

- reducing the costs of the company by giving up the activities that involve creating, producing and placing the advertisement;

- advertising experience of agencies that have employed specialists in many fields of activity and can offer solutions to difficult problems or special solutions;

- creativity of advertising agencies.

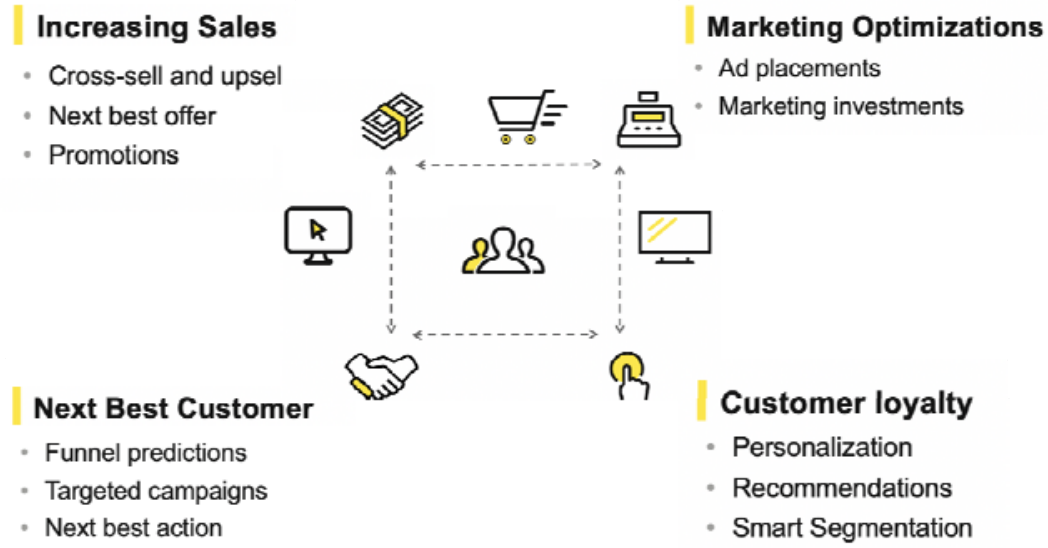

Figure 1. An advertising agency functions

Unlike the company's employees, the advertising agencies look at the products of a company with a greater degree of objectivity and depth and most of the time they have the right ideas and especially the means to put them into practice. There are artificial intelligence tools today that can automatically optimize 
advertising spending and identify key elements that make up the target group's preferences. AI can process the data of an advertising agency, in terms of advertising costs and campaign results, so that they can find out what actions (changes in spending, changes in targeting, etc.) will lead to better performance. This is also valid on a large number of complex campaigns.

In this sense we can exemplify through the data published by an advertising company, which realized a campaign for a gift shop, which used artificial information from Albert to automatically optimize its paid advertising. The results were impressive. [5] Albert has identified ways to optimize spending and target higher revenue from advertising spending and has thus become more efficient than a human expert. The tool using the information it gained from sufficient amounts of advertising spending data, actually found new audiences for the gift shop work that the company didn't even know were relevant customer segments. [5]

We can conclude that AI's ability to learn and improve without human involvement has offered the brand a massive competitive advantage over both human-powered ad programs and those using traditional software. The AI can also specify the performance of the ad agency ads and can even help increase the performance. For this reason, it is used today to substantially improve the activity carried out in advertising campaigns. Advertising platforms, especially Facebook, have AI that helps advertisers create ads and recommendations much faster than the work of advertisers, using content you've already uploaded. Many of the tools available at the moment carry this process forward. Thus, an AI-based Phrasee tool actually writes from scratch Facebook and Instagram ads, and the ads are designed to turn into clicks based on those worked in the past. The tool improves over time by itself, as it learns from each new ad which language leads to better performance.

AI-based tools are also aids in saving the time required for the overall management of advertising campaigns. Thus WordStream uses machine learning to quickly and efficiently analyze your advertising campaigns. The same tool identifies ways to make changes to your Facebook, Google and Bing ad campaigns with a few clicks.

\section{AI for digital marketers}

The IT teams in the advertising agencies were the first to adopt the automatic learning and AI for automatic bidding for digital ads.

A report from August 2019 [6] stated that $65 \%$ of US households have TVs connected to the Internet, which means that the growth of connected devices and smart TVs is significant. With this tendency, AI intervenes to allow more targeted, relevant programming, improving the audience experiences. We can thus identify two examples:

A personalized television powered by machine learning, allows digital marketing to provide specific ads to the right consumers. These marketers target consumers who are more likely to connect with their message based on learned data. This tendency was observed in political election campaigns, when several digital planners targeted television advertisements addressed to viewers who identified with a particular political party.

In non-televised environments, such as websites, brands use AI to interact with consumers to learn more about them and to make smarter recommendations. Both Amazon and many other e-commerce companies use referral systems to talk and make smart recommendations about the products needed by the customer in a given context.

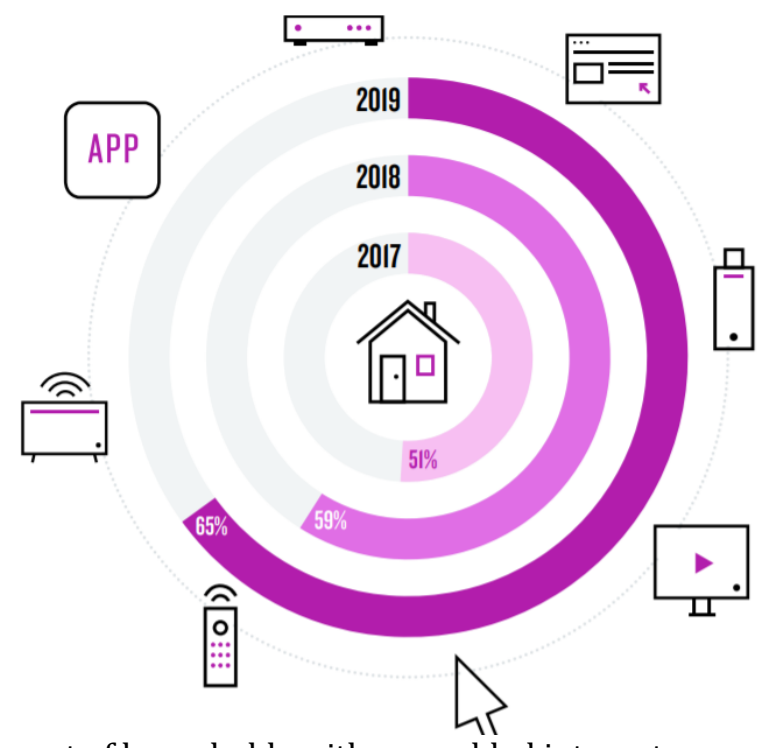

Figure 2. Percent of households with an enabled internet connected device or smart tv Source - https://www.nielsen.com/ 
The most used functionalities of $\mathrm{AI}$ in advertising and marketing are:

- Recommendations - Using predictive analysis, an agency is able to provide better recommendations to their users, allowing them to make the most of the services and products offered. This results in high customer satisfaction and a high value proposition. Similarly, it could combine data from its applications with data from third parties to provide more personalized training to their users.

- Social Semantics - Facebook AI Research has been working in recent years to build one of the most innovative and highly intuitive AI platforms to analyze aerial images of topography and find evidence of human life in order to use the Internet drone to use it sends wireless signals to places where people do not already have Internet access. According to Facebook's AI research, the use of this state-of-the-art technology can be extended beyond internet drones.

- Programmatic advertising - When it comes to ad targeting, displaying the right ads to the right customer at the right time has been a challenge for advertisers when selling ad clips. With the incorporation of artificial intelligence technologies, such as programmatic advertising, many advertising agencies have managed to automate the process of buying and selling advertising space by combining the power of AI and real-time bidding on mobile, video and social channels. This AI technology effectively analyzes a visitor's behavior and enables real-time campaign optimization for an audience that is likely to transform.

- The use of artificial intelligence and machine learning to buy real-time advertising, instead of going through human negotiations and pre-set prices, are the components of programmatic advertising. Programmatic advertising exists in a wide range of digital channels, including display, mobile, video and social. Traditional offline channels are about to become fully digitized. We begin to see that the channels in the house make programmatic advertising through digital screens in bus stations, shopping centers and advertising panels.

- Email Marketing Language - In email marketing, the best way to get more clicks, stimulate open rates and increase responses is to write better emails. However, many advertisers have come up with innovative enterprise email marketing platforms to write better headlines and use customer-focused email marketing to stimulate clicks, open rates and revenue. Their artificial intelligence mechanism learns from customer response values and optimizes the language that forces customers to respond to your emails.

\section{Predictive Analysis}

For several years, companies have used advanced analytical tools to detect fraud, assess risk and even predict or make different forecasts or diagnoses. However, understanding the results of predictive analysis and transforming data into intuition was not always easy and usually required advanced skills. Limited and hard-to-reach computing capabilities, predictive technologies have never been used in marketing strategies. Today, AI-based marketing tools - artificial intelligence - chatbots, dynamic pricing models, etc. they are both easy to access and accessible, creating opportunities for more companies to take advantage of the benefits of global analysis, even on a lower budget.

Predictive analysis is "using data, statistical algorithms and machine learning techniques to identify the likelihood of future results based on historical data. The purpose is to know what happened to provide the best assessment of what will happen in the future. But predictive marketing uses data science to accurately predict which marketing actions and strategies are most likely to succeed. In short, predictive intelligence drives marketing decisions. Thus 2 questions appear

- how can predictive analytics add more clarity and clarity to your marketing decisions?

- how can big data analytics help businesses achieve value?

Companies connected to the future already want to know more than just what has happened in the past; they want to use their data to predict the future. Predictive analysis already makes current and historical data more valuable, helping you better understand relationships to make more informed decisions. Today, "91\% of top marketers are fully dedicated or already implementing predictive marketing." They more accurately predict customer behavior. For marketers, customer information is the name of the game. The more you understand your customers - what they want, when they want it and how - the easier it is to reach them through the right messaging channels at the right time.

Using guided predictive techniques of machine learning and artificial intelligence, predictive modeling helps evaluate future customer behaviors by identifying patterns and similarities between variables in the data. For example, the regression analysis identifies correlations between customers' previous purchasing behaviors to determine the likelihood of future purchases.

Predictive models can help eliminate unsatisfied customers who risk losing, as well as identify happy customers who may be ready to buy. Running customer data through predictive models can help you better anticipate your behavior to better inform your marketing strategy. 


\section{The stages of predictive analytics}

Each advertising campaign involves a unique data analysis phase, not only because of the high level of refinement, but also because of the distinct data sets that each company provides as input. However, most go through the following workflow:

- Define the campaign - This stage refers to determining all the essential components of the campaign. It is essential to establish the expected results and results, the input and available data resources and the roles needed to execute the implementation. As a result of this step, a company should clearly understand what it hopes to achieve when the project is completed.

- Data collection - At this stage, companies gather all the data they consider useful as contributions to their projects. Since data processing is the essence of predictive analytics, choosing the right types, purposes and sources of data is essential for the positive outcome. For example, if a campaign aims to improve business segmentation, it must work on demographic and behavioral data collected from a variety of sources. These sources include traditional tools for collecting customer information, such as CRM systems, face-to-face interactions or telephone engagements, as well as conversations on social media channels, laptops and other IT devices. It is important to know that most of the data is collected and stored in a raw, unprocessed state.

- Data processing - The correct data must be provided before obtaining any information. Data processing is a stage that requires a lot of resources, but that can have the greatest impact on the results of the campaign. The data collected at the previous stage is usually quite chaotic, some can be structured (for example, in a table), but most of the time they are unstructured, missing some attributes. Most of the time the data is unstructured and in different formats (audio, video, social media). Such unstructured data is difficult to process for AI and therefore requires some sorting, cleaning or sorting before algorithms can use them. In the context of a marketing campaign, it is of crucial importance to ensure strict compliance with data protection laws when processing personal or sensitive information.

- Modeling - Modeling is a central part of the process of creating and extending data models that define how an organization should collect, update and store data. The models are used to understand the logic in the data and to make predictions based on the conclusions drawn. You can imagine them as abstract graphs that organize data sets and determine the relationships between them. They range from linear models that work with two correlated variables to complex neural networks of machine learning that are able to independently process large volumes of data to identify and deduce subtle correlations.

- Interpretation - Once different algorithms and statistical models have processed data, the results can be interpreted and presented to answer campaign target business questions. Visualization is applied to ensure a better understanding of predictions. Finally, the information obtained should be translated into actions to be implemented and incorporated into strategic marketing planning.

\section{Predictive analysis models}

- Classification model - The classification model is the simplest of several types of predictive analysis models. The classification model divides the data into categories based on what it learns from historical data. Classification models are best for answering yes or no to questions, providing a broad analysis that is useful for guiding decisive actions.

- Clustering Model - The clustering model sorts the data into separate intelligent groups, nested based on similar attributes. Using the clustering model, firms can quickly separate customers into similar groups based on common characteristics and develop strategies for each group on a larger scale.

- Forecast model - One of the most used predictive analysis models, the forecast model deals with the prediction of the metric values, estimating the numerical value for the new data based on the learning from the historical data. This model can be applied wherever historical numerical data is available.

- Outliers Model - The outliers model is oriented around the abnormal data entries in a data set. It can identify anomalous numbers either alone or together with other numbers and categories. The recording of a peak could indicate an unplanned failure, fraud, or imminent stop sign. The model is particularly useful for such analysis especially in retail and finance. For example, when we identify fraudulent transactions, the model can evaluate not only the amount, but also the location, time, purchase history and nature of a purchase.

- Time series model - The time series model comprises a sequence of captured data points, using time as the input parameter. Uses the last year of data to develop a numerical value and predicts the next three to six weeks of data using this measurement. Thus, if the owner of a restaurant wants to predict how many people are likely to visit his business, he could use the gross method of calculating the average number of visitors in the last 30 days. However, growth is not always static or linear, and the time series model can better model exponential growth and better align the model to the trend of a firm.

\section{Conclusions}

It is almost impossible to imagine all the applications for AI in the advertising industry, because every day progress is being made to improve our lives. Sometimes we use AI-powered tools and don't even 
realize it. If we were thinking of any new technological solution we tried or came face to face lately - is it powered by artificial intelligence?

Those in the advertising industry do not benefit from remaining passive in the technological evolution, and the only option that will keep them on the market is to remain curious about the new tools developed and their usefulness in the advertising campaigns. And, to consider how the technology will help the consumer, as well as the implications on how AI might affect their privacy and data security.

With AI and machine learning, we have access to information that traditionally we could not process at a higher level. As we continue to create links between AI information with human experience, the advertising industry will only become stronger and more efficient.

\section{Acknowledgement}

This work was supported by a grant of the Romanian Ministry of Research and Innovation, CCCDI - UEFISCDI, project number PN-III-P1-1.2-PCCDI-2017-0800 / 86PCCDI/2018 - FutureWeb, within PNCDI III.

\section{References}

1. Dubois, Pierre - Jolibert, Alain Louis - Marketing (teorie si practica), Vol.II, Editura Economica, Bucuresti, 1994.

2. Folteanu, Florin, Ladar, Lucian (coordonatori) - Marketing, Editura Brumar, Timisoara, 2001

3. https://www.marketingaiinstitute.com/blog/how-to-use-artificial-intelligence-in-advertising

4. http://attend.it.uts.edu.au/icita05/International Conference on InforTechnology for Application (ICITA 2004)

5. https://www.marketingaiinstitute.com/blog/how-one-ecommerce-company-used-ai-to-get-a-3000-return-on-adspend?_ga=2.72904912.2134330631.1569846823-1786261508.1548863773

6. https://www.nielsen.com/wp-content/uploads/sites/3/2019/08/local-watch-report-aug-2019.pdf

7. https://www.cnbc.com/video/2016/10/13/making-ads-smarter-ai-in-advertising.html 